\title{
Triggering on B-Jets at CDF II
}

\author{
Silvia Amerio, Massimo Casarsa, Giorgio Cortiana, Julien Donini, Donatella Lucchesi, and Simone Pagan Griso
}

\begin{abstract}
In this paper we present a trigger algorithm able to select online events enriched of $b$-jets. This feature is of central interest in order to extend the physics reach for Standard Model and Minimal Super Symmetric Model Higgs decaying into a pair of $b$-quarks. The algorithm fully exploits the recently upgraded CDFII tracking system and Level 2 CALorimeter cluster finder. These upgrades are necessary to cope with Tevatron increasing luminosity and provide new and refined trigger primitives that are the key elements of our algorithm together with the already existing Silicon Vertex Trigger. A $b$-hadron can travel some millimeters before decaying and the trigger algorithm exploits this characteristic by searching for tracks displaced with respect to the primary vertex and matched to energetic jets of particles. We discuss the study and the optimization of the algorithm, its technical implementation as well as its performance. The new trigger provides an efficient selection for Higgs decaying into a pair of $b$-quarks and runs up to high luminosity with an acceptable occupancy of the available bandwidth.
\end{abstract}

Index Terms- $b$-tag, CDF, Higgs boson, tevatron, trigger.

\section{INTRODUCTION}

A great effort is currently underway in experimental particle physics to discover or exclude the Higgs boson, whose existence could explain the dynamics of electroweak symmetry breaking and the origin of particle masses [1], [11]. Standard Model (SM) theory predicts the existence of one neutral Higgs boson whose dominant decay mode, for masses lower than $135 \mathrm{GeV}$, is in pairs of $b$-quarks. Theories beyond the Standard Model, such as the Minimal Supersymmetric Standard Model extension (MSSM) [2], predict the existence

Manuscript received December 17, 2008; revised February 26, 2009. Current version published June 17, 2009. This work was supported by the U.S. Department of Energy and National Science Foundation; the Italian Istituto Nazionale di Fisica Nucleare; the Ministry of Education, Culture, Sports, Science and Technology of Japan; the Natural Sciences and Engineering Research Council of Canada; the National Science Council of the Republic of China; the Swiss National Science Foundation; the A.P. Sloan Foundation; the Bundesministerium für Bildung und Forschung, Germany; the Korean Science and Engineering Foundation and the Korean Research Foundation; the Particle Physics and Astronomy Research Council and the Royal Society, UK; the Institut National de Physique Nucleaire et Physique des Particules/CNRS; the Russian Foundation for Basic Research; the Comisión Interministerial de Ciencia y Tecnología, Spain; the European Community's Human Potential Programme; the Slovak R\&D Agency; and the Academy of Finland.

S. Amerio, D. Lucchesi, and S. Pagan Griso are with the University of Padova, Padova, Italy and also with INFN, Padova, Italy (e-mail: amerio@pd.infn.it; lucchesi@pd.infn.it; simone.pagan@pd.infn.it).

M. Casarsa is with Fermilab, Batavia, IL 60510-5011 USA (e-mail: casarsa@fnal.gov).

G. Cortiana was with the University of Padova, Padova, Italy and INFN, Padova, Italy. He is now with Max-Planck-Institut fuer Physik, Muenchen, Germany (e-mail: cortiana@mppmu.mpg.de).

J. Donini was with the University of Padova, Padova, Italy, and INFN, Padova, Italy. He is now with LPSC Grenoble CNRS/IN2P3, Grenoble, France (e-mail: julien.donini@1psc.in2p3.fr).

Digital Object Identifier 10.1109/TNS.2009.2020405 of three neutral Higgs particles with production cross sections that can be significantly enhanced depending on the MSSM parameter space. The dominant decay mode for MSSM neutral Higgs is in pairs of $b$-quarks, with a branching ratio of $\sim 90 \%$.

The identification of jets originating from $b$-quarks is thus of central interest for any search of a neutral Higgs boson and the possibility to select $b$-jets online could greatly improve the purity of the collected samples.

Since Tevatron luminosity has increased above $1.5 \times$ $10^{32} \mathrm{~cm}^{-2} \mathrm{~s}^{-1}$ the existing $b$-jet triggers suffer from very high rates and thus have to be prescaled and/or enabled only when the luminosity goes below a given threshold. This way of keeping rate under control yields big drawbacks in terms of efficiency losses on signal events. The new trigger algorithm described here is able to select events with $b$-jets up to luminosity of $3.0 \times 10^{32} \mathrm{~cm}^{-2} \mathrm{~s}^{-1}$ and keeps high efficiency on the most desirable signals.

This trigger is optimized for the collection of $H \rightarrow b \bar{b}$ events but aims at having a good efficiency also with respect to the $Z \rightarrow$ $b \bar{b}$ process. The latter is very important for $b$-jet energy scale and resolution determination and as a normalization channel for inclusive $H \rightarrow b \bar{b}$ searches.

In the following we will first review the CDF II detector, its trigger system and the upgrades for high luminosity data taking. We will then illustrate the architecture and the performance of a new $b$-jet trigger optimized to have high efficiency for $H \rightarrow b \bar{b}$ events while maintaining acceptable rate at high luminosity.

\section{CDF II DETECTOR}

CDF II is a general-purpose, azimuthally and forward-backward symmetric detector located at the Tevatron $p \bar{p}$ collider at Fermilab. It consists of a charged-particle tracking system immersed in a 1.4 T magnetic field followed by calorimeters which are surrounded by muon detectors. A detailed description can be found in [3].

The CDF II coordinate system uses $\theta$ and $\phi$ as the polar and azimuthal angles respectively, defined with respect to the proton beam axis direction, $z$. The pseudo-rapidity $\eta$ is defined as $\eta \equiv$ $-\ln [\tan (\theta / 2)]$. The transverse momentum of a particle is $p_{T}=$ $p \sin \theta$ and the transverse energy is defined as $E_{T}=E \sin \theta$.

The detector parts relevant for our trigger studies are the tracking and calorimetric systems. Charged particle trajectories are detected by a 8-layers silicon microstrip detector and a drift chamber which provide $|\eta|$ coverage up to 2.0 and 1.0 respectively. The drift chamber consists of cells divided into 8 super-layers, each containing 12 layers of sense wires. The odd superlayers have wires parallel to the beam axis (axial layers) while the even ones have wires tilted by $2^{\circ}$ (stereo layers) in order to provide stereo information. The calorimeters are used 
to measure electromagnetic showers and jets from quark fragmentation and consist of projective towers with electromagnetic and hadronic sections covering the region up to $|\eta|<3.6$.

\section{CDF II TRIGGER SYSTEM}

The CDF II trigger system has a three level architecture designed to reduce the amount of data from $2.53 \mathrm{MHz}$, the bunch crossing rate, to approximately $150 \mathrm{~Hz}$ to be written on tape. At Level-1 (L1) raw muons, tracks and calorimeter information are processed to produce a $\mathrm{L} 1$ decision. $\mathrm{L} 1$ is a synchronous 40 stages pipeline and it is based on custom-designed hardware. It can provide a trigger decision in $5.5 \mu \mathrm{s}$ and during normal data taking its output rate is typically below $30 \mathrm{kHz}$. When an event is accepted at L1, subsets of detector information are sent to the Level-2 (L2) system, where some limited event reconstruction is performed and a decision is taken. The L2 is an asynchronous pipeline and it is based on a combination of custom-designed hardware and commodity processors. Its average latency is $20 \mu \mathrm{s}$ and its maximum output rate is $1 \mathrm{kHz}$. Upon L2 accept, the full detector data is readout and sent to Level-3 (L3) processors farm for further processing. Events accepted at L3 are sent to mass storage.

Recently, in order to sustain higher trigger rates due to Tevatron increasing luminosity, many subsystems of the CDF II trigger system had to be upgraded. The upgrades involved the L1 tracking processor (XFT, eXtremely Fast Tracker) and the Level 2 CALorimeter (L2CAL) cluster finder. The new system provides new tools that can be used to design innovative trigger algorithms and helps to keep trigger rates under control.

These upgrades, combined with the existing Silicon Vertex Trigger (SVT), are used for the implementation of the online $b$-tagging algorithm.

\section{A. XFT Upgrade}

XFT [4], [5] has been developed to reconstruct tracks in the plane of the drift chamber transverse to the beam axis in time for L1 decision using hit data from the 4 axial superlayers of the chamber. Track identification is performed searching and combining track segments in the 4 axial superlayers of the drift chamber. XFT measures transverse momentum and azimuthal angle $\phi$ of all the tracks with $p_{T}>1.5 \mathrm{GeV} / \mathrm{c}$ with an efficiency greater than $96 \%$ and a resolution $\sigma_{p_{T}} / p_{T}^{2} \sim 2 \%\left(\mathrm{GeV}^{-1}\right)$ and $\sigma_{\phi} \sim 6 \mathrm{mrad}$.

The upgraded XFT maintains the existing axial system and new boards are added to find track segments also in the outer stereo layers of the chamber. The upgraded system can now reject at L1 fake axial tracks by requiring the association with stereo segments with a rejection factor of about 7 . An axial track associated to segments in the stereo layers is a stereo confirmed track. Moreover, stereo segments can be sent to L2 and matched to the axial tracks for 3D-track reconstruction which provides a good resolution on $\cot \theta\left(\sigma_{\cot \theta}=0.11\right)$ and $z_{0}\left(\sigma_{z_{0}}=11 \mathrm{~cm}\right)$.

\section{B. L2CAL Upgrade}

The old L2 calorimeter trigger algorithm was based on clusters formed by simply combining contiguous regions of trigger towers with an energy deposition above a given threshold in the electromagnetic and the hadronic calorimeters. At high luminosity, when multiple proton-antiproton interactions occur in the same bunch crossing, calorimeter occupancy is increased and it can happen that clusters produced by different particles are merged together, yielding a high L2 accept rate due to fake clusters above threshold. Moreover, due to intrinsic hardware limitation, the old system used only 8-bit energy resolution even if 10-bit trigger tower information was available.

Now the upgraded system [6], [7] uses a fixed cone cluster finding algorithm which prevents fake cluster formation and exploits the full trigger tower energy information. A jet is formed starting from a seed tower above a threshold and adding all the towers inside a fixed cone centered at the seed tower and having a radius $\Delta R=\sqrt{\Delta \phi^{2}+\Delta \eta^{2}}=0.7$ units in the azimuthpseudorapidity space. The jet position is calculated weighting each tower inside the cone according to its transverse energy. This upgrade has reduced L2 trigger rate and has provided at L2 jets with a quality nearly equivalent to offline ones.

\section{SVT}

SVT [8], [9] is a L2 trigger processor dedicated to the reconstruction of charged particle trajectories in the plane transverse to the beam line. SVT combines hits from silicon detectors with tracks reconstructed by XFT. The association is performed by an associative memory, a massive parallel mechanism based on the search of coincidences between hits in silicon detectors and XFT tracks. When such an association is found, a track fitter performs quality cuts and estimates track parameters using the full available spatial resolution in a linearized fit. Overall SVT tracking efficiency is about $80 \%$. SVT provides precise measurement of track impact parameter $\left(d_{0}\right)$, curvature and azimuthal angle. Impact parameter is measured with a resolution of $35 \mu \mathrm{m}$ for $2 \mathrm{GeV} / \mathrm{c}$ tracks, which is comparable to the resolution obtained for offline reconstruction.

\section{TRIGger ARChitecture}

The XFT improved tracking capabilities described above and the already available SVT tracker can be combined with improved jet reconstruction in order to perform at L2 an efficient track-jet matching, key element of our $b$-tagging algorithm.

The algorithm is optimized for $H \rightarrow b \bar{b}$ search but it can also be used to collect any final states with $b$-jets and in particular $Z \rightarrow b \bar{b}$ events. Since $b$-quark travels some millimeters before decaying, few tracks in these jets will be displaced from the primary vertex. The idea at the basis of our algorithm is to exploit the displacement of $b$-jet tracks while trying to keep the cut on the jet energies as low as possible in order to limit the bias on dijet invariant mass distribution.

The effect of the trigger on signal events is studied using Monte Carlo generated events: SM Higgs produced via gluon fusion and MSSM Higgs produced in association with a $b$-quark ( $b H$ in the following). In both samples the Higgs has a mass of $M_{H}=120 \mathrm{GeV} / \mathrm{c}^{2}$ and it is forced to decay into a pair of $b$-quarks. In addition a $Z \rightarrow b \bar{b}$ sample has been used to evaluate the effect on lower mass resonances. The rejection of background events, mainly composed by light quark jets, and the trigger bandwidth occupancy are estimated using data events 
collected by a control trigger with the minimal requirement of at least one calorimetric trigger tower above $5 \mathrm{GeV}$ at L1.

\section{A. Level-1}

Given the event topology we aim at selecting, at L1 we require at least two central $(|\eta|<1.5)$ calorimetric towers with $E_{T} \geq 5 \mathrm{GeV}$ and two XFT, stereo confirmed, tracks having $p_{T}>2 \mathrm{GeV} / \mathrm{c}$. These requirements are highly efficient on signal events while providing a significant background reduction. These cuts are used also by other trigger algorithms at CDF II and therefore the L1 trigger bandwidth occupancy is not increased.

\section{B. Level-2}

The core of the new algorithm is at $\mathrm{L} 2$, where the $b$-tagging is performed. The basic idea is to look for displaced tracks in jets and then make additional selections based on variables distinctive of tracks coming from $b$-hadrons. In the following we will describe in more detail the trigger objects and the variables used by the algorithm.

1) Jets: At L2 jets are reconstructed using the fixed-cone algorithm previously described. The radius of the cone in the $\eta-\phi$ plane is 0.7. Jets from Higgs decay tend to be central and energetic $\left(\left\langle E_{T}\right\rangle \sim 40 \mathrm{GeV}\right.$, for $\left.M_{H}=120 \mathrm{GeV}\right)$. However, in order to avoid sizeable bias on the dijet invariant mass distribution, the cut on jet energy is kept as low as possible $(15 \mathrm{GeV})$. Since we require at least one jet matching two XFT tracks, jets have to be in the coverage of the drift chamber $(|\eta|<1.0)$.

2) Tracks: At L2 we have at our disposal tracks reconstructed by SVT as well as by XFT from L1. The first ones carry precise information on azimuthal angle $\phi$, impact parameter $d_{0}$ and curvature $c$. The XFT tracks if stereo confirmed can be subjected to stereo reconstruction, and carry additional information about the track polar angle $\theta$ and its $z_{0}$ position. It is then possible to look for 3D matches between jets and tracks with a precision similar to the offline one.

We require at least two XFT stereo reconstructed tracks per event and both matched to one of the jets in $\eta-\phi$ plane $(\Delta R<$ $0.7)$.

3) Signed Impact Parameter: Since tracks from $b$-hadrons are likely displaced with respect to the primary vertex, the track $d_{0}$ is a good discriminating variable. Its discriminating power can be enhanced if we assign it a negative or positive sign depending on the point of intersection of the track with the jet axis. The sign is positive if this point is in the same hemisphere of the jet's direction, negative otherwise. In Fig. 1 signed $d_{0}$ distribution is shown for $H \rightarrow b \bar{b}$ signal and light quarks jet background. Tracks from $b$-jets tend to have positive values of the signed $d_{0}$ with respect to background.

4) b Decay Length: The following relation between impact parameter $d_{0}$ and azimuthal angle $\phi$ holds for tracks coming from $b$-hadron decays:

$$
d_{0}=R_{b} \sin \left(\phi_{b}-\phi\right) \sim R_{b}\left(\phi_{b}-\phi\right)
$$

where $R_{b}$ and $\phi_{b}$ are $b$-quark decay length and azimuthal angle respectively. The approximation holds when the $b$-hadron decay tracks are collimated along the b-hadron momentum direction.

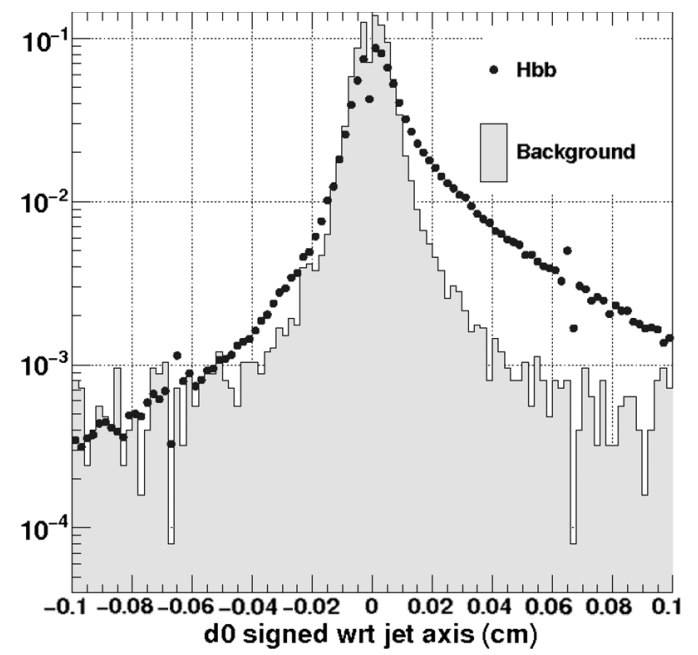

Fig. 1. Signed impact parameter distribution for tracks matching L2 jets.

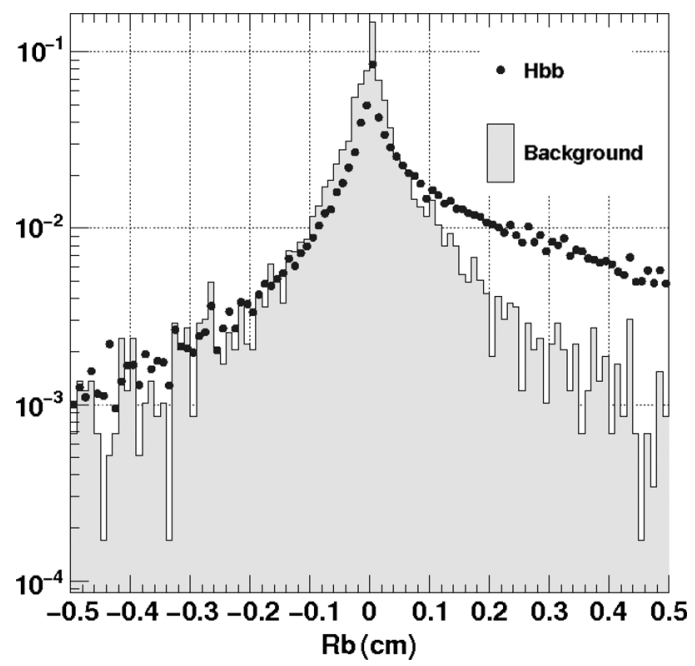

Fig. 2. $b$-quark decay length $\left(R_{b}\right)$ distribution for tracks matching L2 jets.

Real $b$-jets have a decay length distribution predominantly at positive values (Fig. 2), thus it is possible to discriminate them from light quark background by using it.

\section{Level-2 Cut Optimization}

The optimization of the cut on $R_{b}$ and signed $d_{0}$ is performed aiming at the best compromise between efficiency for $H \rightarrow b \bar{b}$ signal and background rejection. In order to keep under control the L2 output rate, we need to cut on $d_{0}$ of both tracks matched to one of the jets.

For a given cut on $R_{b}$, we measure the efficiency on signal and on background for different cuts on signed $d_{0}$ obtaining the family of curves in Fig. 3. Each curve represents a cut on $R_{b}$ and each point on the curve is a different cut on $d_{0}$, for $d_{0}$ ranging from $-250 \mu \mathrm{m}$ to $250 \mu \mathrm{m}$. We choose to cut on $R_{b}>0.1 \mathrm{~cm}$. This value provides efficiency for signal (background) ranging from $32 \%$ to $6 \%(0.2 \%$ to $0.01 \%)$ depending on $d_{0}$ cut.

Once $R_{b}$ is fixed, we look at the efficiency for signal and background varying the cut on $d_{0}$ (Fig. 4). A good compromise between signal efficiency and background rejection is $d_{0}>90 \mu \mathrm{m}$ 


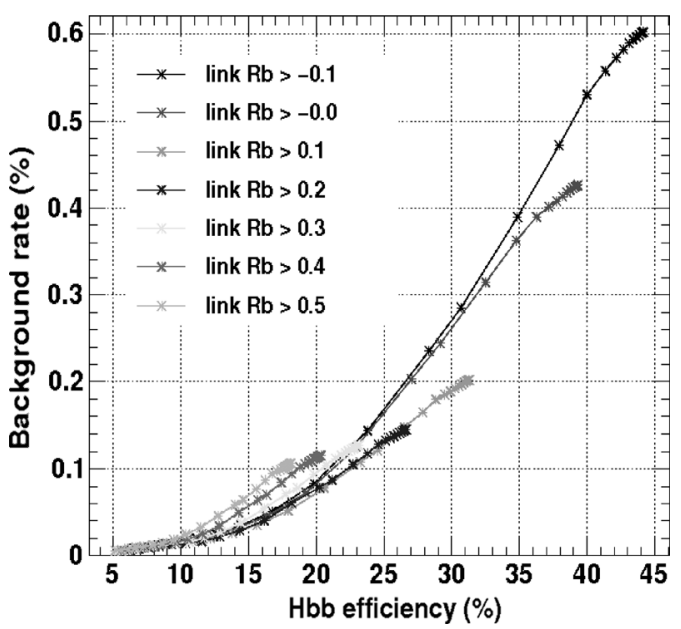

Fig. 3. L2 cut optimization: scan of signed $d_{0}$ values for different values of $R_{b}$.

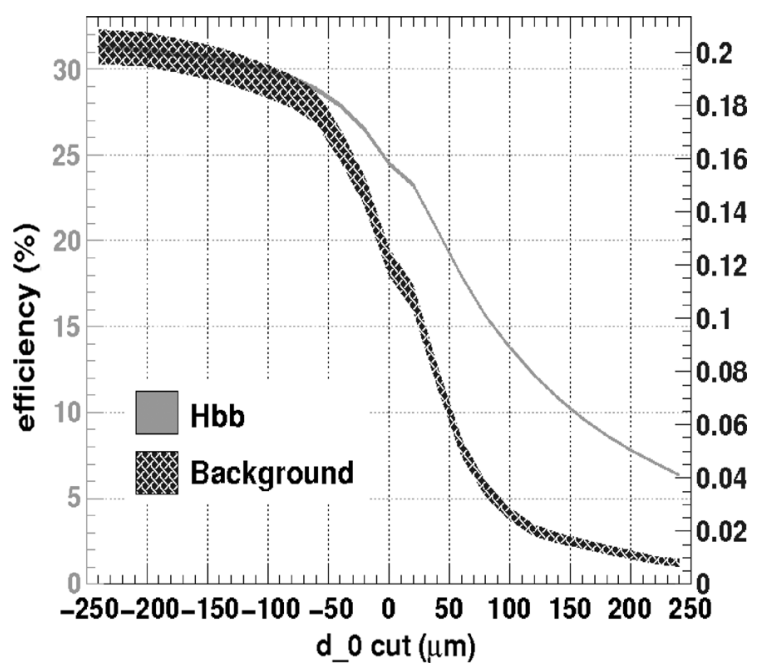

Fig. 4. Efficiency of the cut on signed $d_{0}$ for signal (left-hand vertical scale) and background (right-hand vertical scale) events. Both curves are for $R_{b}>$ $0.1 \mathrm{~cm}$.

which provides $14 \%$ efficiency for $H \rightarrow b \bar{b}$ events and only $0.03 \%$ efficiency for background.

\section{Level-3}

In order to create a $\mathrm{L} 3$ trigger algorithm highly efficient on signal events, we simply confirm L2 requirements using L3 offline variables.

We require two central $(|\eta|<1.0)$ jets $(\Delta R=0.7)$ having $E_{T} \geq 15 \mathrm{GeV}$. Simultaneously we require two tracks reconstructed using information from the drift chamber only and matched in azimuthal angle and curvature to two SVT tracks to get information on impact parameter.

The match is done using the azimuthal angles and the curvatures as measured by the two tracking systems by requiring $\Delta \phi \leq 0.015 \mathrm{rad}$ and $\Delta c \leq 0.00015 \mathrm{~cm}^{-1}$. To keep L3 requirements as simple as possible and to exploit existing CDF II L3 trigger modules, we do not require the match between the two tracks and one of the jets. For the same reason, we do not consider the impact parameter signed with respect to the jet axis and simply require that both SVT tracks have $\left|d_{0}\right|>90 \mu \mathrm{m}$.

\section{SUMmary OF TRIGGer REQUiREMENTS}

In the following we summarize the requirements of the new trigger algorithm:

\section{A. Level-1}

- Two central $(|\eta|<1.5) E_{T} \geq 5 \mathrm{GeV}$ calorimetric towers.

- Two XFT tracks having $p_{T}>2 \mathrm{GeV}$.

\section{B. Level-2}

- Two jets with $E_{T} \geq 15 \mathrm{GeV}$ and $|\eta|<1.0$.

- Two XFT-SVT tracks matched to one of the leading jets - forming a 2-track displaced vertex with $R_{b}>0.1 \mathrm{~cm}$, — having signed $d_{0}>90 \mu \mathrm{m}$.

C. Level-3

- Two jets with $E_{T} \geq 15 \mathrm{GeV}$ and $|\eta|<1.0$.

- Two tracks reconstructed using information from the drift chamber and matched to two SVT tracks with $\left|d_{0}\right|>90 \mu \mathrm{m}$.

\section{TRIGGER IMPLEMENTATION}

As far as the trigger implementation is concerned, the main issue is at L2 where the track-jet match is performed and strict timing requirements have to be fulfilled. Since the average latency at L2 is only $\sim 20 \mu \mathrm{s}$ and XFT 3D track reconstruction algorithm needs about $2 \mu$ s per track, the order of the algorithm requirements is important. At least two jets and two SVT tracks passing the required characteristics (in $E_{T}, \eta$ for the jets, signed $d_{0}$ and $R_{b}$ for the tracks) and matched in azimuthal angle $(\Delta \phi<0.7)$ must be present. Only if the event satisfies these selections we perform XFT 3D reconstruction and require the 3D match between the two tracks and one of the jets. Our algorithm employs in average $5.3 \mu$ s to perform a complete selection, well below L2 average latency.

\section{TRIGGER PERFormanCE}

\section{A. Bandwidth Occupancy}

The most important requirement of the new algorithm is the capability to be used in the trigger up to high luminosity $(\mathrm{L}=$ $3 \times 10^{32} \mathrm{~cm}^{-2} \mathrm{~s}^{-1}$ ) without saturating its bandwidth. The trigger cross section or the rate as a function of the instantaneous luminosity are used to evaluate the bandwidth occupancy since they are related according to Rate $=\sigma_{\text {Trig }} \mathcal{L}$, where $\mathcal{L}$ is the instantaneous luminosity and $\sigma_{T r i g}$ is the trigger cross section which in general depends on luminosity.

For each trigger level we monitor the cross section as a function of the instantaneous luminosity (Figs. 5-7). The cross section of our trigger is represented by the light grey points, to be compared with the simulation obtained on the control trigger data (triangles). The value at $\mathrm{L}=3 \times 10^{32} \mathrm{~cm}^{-2} \mathrm{~s}^{-1}$ is extrapolated fitting the measured cross section up to $2.0 \times 10^{32} \mathrm{~cm}^{-2} \mathrm{~s}^{-1}$. We use a second order polynomial 


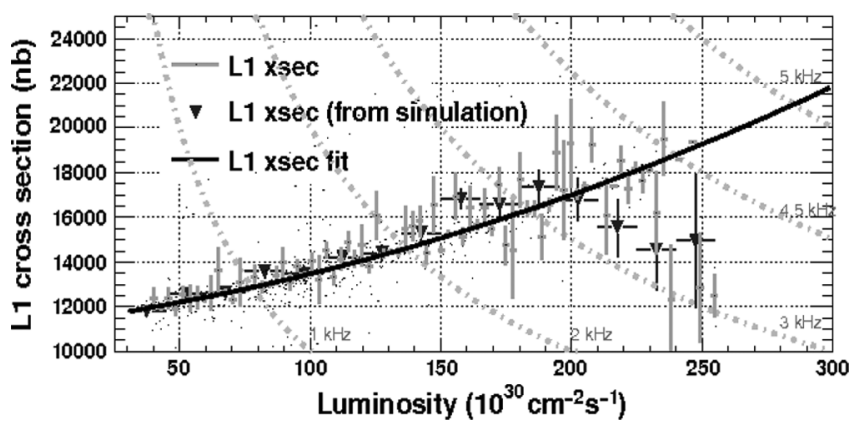

Fig. 5. L1 cross section as a function of instantaneous luminosity.

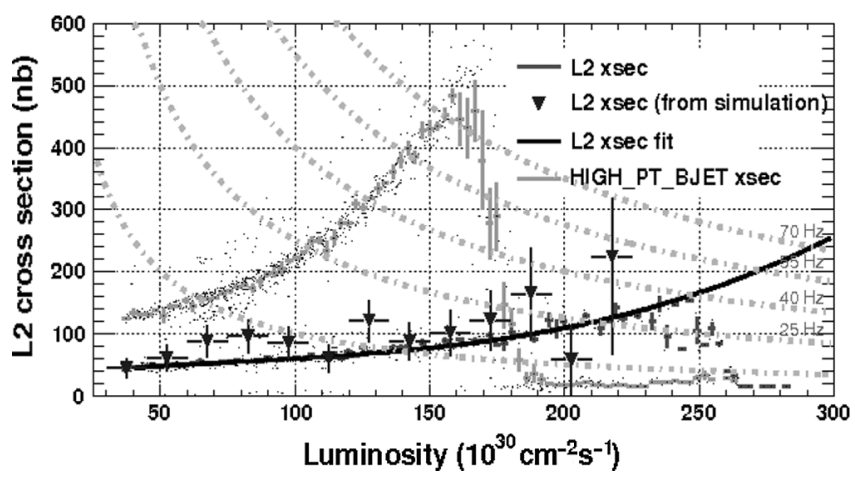

Fig. 6. L2 cross section as a function of instantaneous luminosity.

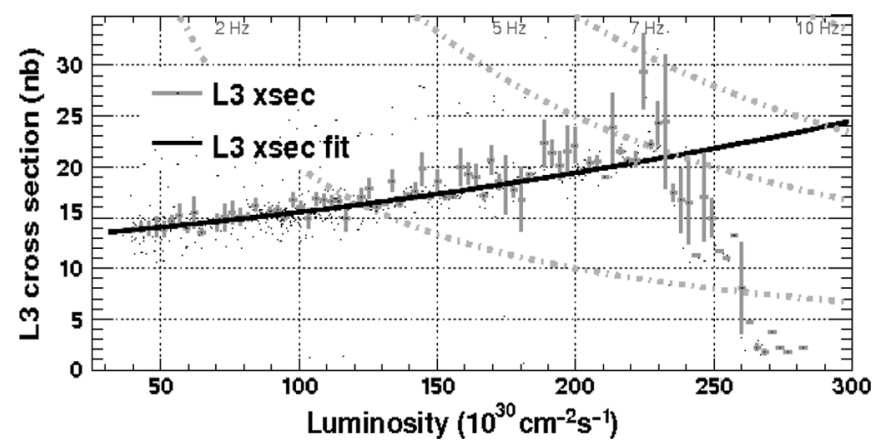

Fig. 7. L3 cross section as a function of instantaneous luminosity.

function for L1 and L3 and a third order polynomial for L2. The dashed curves represent the cross section as a function of the instantaneous luminosity for fixed output frequencies.

L1 overall maximum output rate is $\sim 30 \mathrm{kHz}$. Our trigger has a cross section of $\sim 22000 \mathrm{nb}$ at $3 \times 10^{32} \mathrm{~cm}^{-2} \mathrm{~s}^{-1}$, which corresponds to approximately $6600 \mathrm{~Hz}$. The points decreasing over $\mathrm{L}=2.3 \times 10^{32} \mathrm{~cm}^{-2} \mathrm{~S}^{-1}$ are due to a temporary prescale factor applied at L1.

At L2, where the maximum accept rate must remain below $1 \mathrm{kHz}$, the extrapolated cross section, at the highest luminosity, is $\sim 240 \mathrm{nb}$ corresponding to $\sim 70 \mathrm{~Hz}$. Superimposed is the cross section of the HighPtBJet trigger, used before the completion of the CDF II trigger upgrade to select $b \bar{b}$ final states [10]. This trigger had to be heavily prescaled above the luminosity of $1.6 \times 10^{32} \mathrm{~cm}^{-2} \mathrm{~s}^{-1}$ in order to avoid the saturation of the L2 bandwidth.
TABLE I

Trigger Performance: L1, L2 AND L3 TRIGGer EFFICIENCIES

\begin{tabular}{|c|c|c|c|}
\hline & $H \rightarrow b b$ & $b H$ & $Z \rightarrow b b$ \\
\hline L1 Eff (\%) & 70 & 69 & 38 \\
L2 Eff (\%) & 14 & 43 & 6 \\
L3 Eff (\%) & 13 & 11 & 5 \\
\hline
\end{tabular}
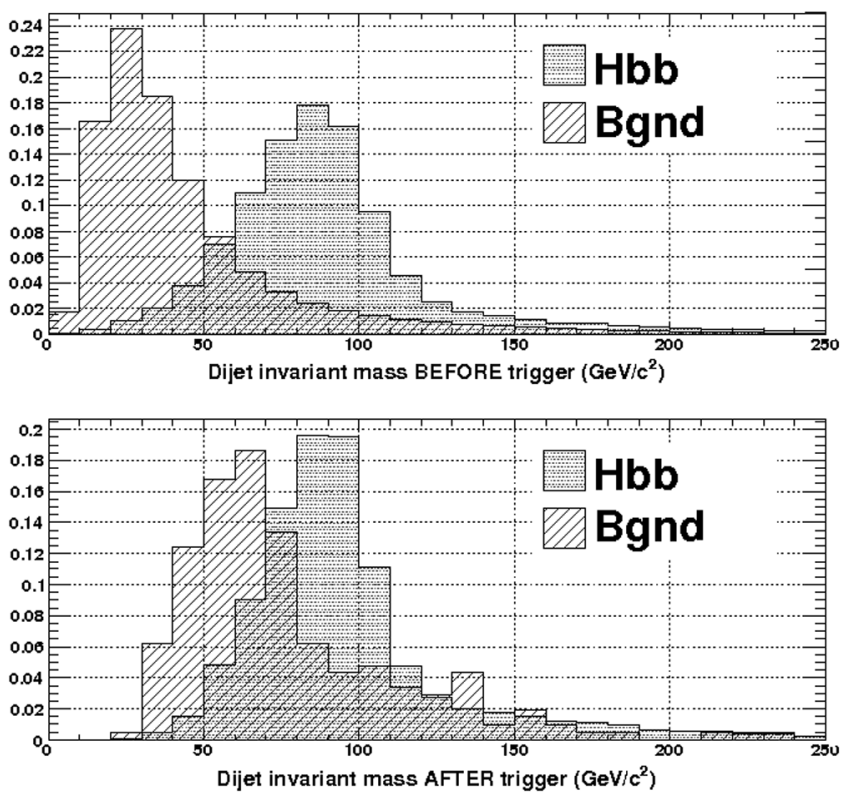

Fig. 8. Dijet invariant mass distribution (raw jet energies) for signal and background events before and after trigger application.

Finally L3 has a predicted cross section of $25 \mathrm{nb}$ giving an output rate of $\sim 7 \mathrm{~Hz}$, well below the $\mathrm{L} 3$ maximum output rate of $150 \mathrm{~Hz}$. For L3 no estimate from the simulation is available, because of low statistic of the control trigger data sample.

\section{B. Efficiency for Signal Events}

The overall efficiency of the trigger (Table I) is $13 \%$ for SM $H \rightarrow b \bar{b}, 11 \%$ for MSSM $b H$ and $5 \%$ for $Z \rightarrow b \bar{b}$ events. These values are similar to the efficiencies provided by the HighPtBJet trigger, but as the new trigger algorithm can run up to higher instantaneous luminosity without being prescaled it provides an overall gain of $40 \%$ on signal acceptance.

\section{Effect on Dijet Invariant Mass}

The effect of the trigger selections on the dijet invariant mass distribution is shown in Fig. 8. The top plot shows signal and background distributions both normalized to one before any trigger requirement, while the bottom plot shows the same distributions after L2. The invariant mass is calculated using raw jet energies. The signal peak is left almost unchanged by trigger requirements while the background distribution, as expected, is shifted towards higher values but still separated from the signal.

\section{CONCLUSIONS}

We have developed and implemented a trigger algorithm capable of selecting samples of events enriched of $b$-jets. The al- 
gorithm exploits improved track and jet reconstruction available from recent trigger upgrades. It is based on track-jet matching at L2 with loose requirements on jet energies, in order to minimize the effect on dijet invariant mass distribution. The trigger performance have been studied using Monte Carlo generated signal samples and data collected by a control trigger path. The algorithm has a good efficiency on signal events and a satisfactory effect on dijet invariant mass while keeping the rate low as required. The new trigger has been officially implemented in the data taking since Spring 2008: it successfully meets the CDF II trigger strict timing requirements and it can run up to high instantaneous luminosity with an acceptable bandwidth occupancy. So far more than $500 \mathrm{pb}^{-1}$ have been collected by this trigger and are ready to be analyzed. We expect that more than $2 \mathrm{fb}^{-1}$ will be available by the end of 2010 .

\section{ACKNOWLEDGMENT}

The authors would like to thank the Fermilab staff and the technical staffs of the participating institutions for their vital contributions.

\section{REFERENCES}

[1] P. W. Higgs, "Broken symmetries, massless particles and gauge fields," Phys. Lett., vol. 12, p. 132, 1964.

[2] D. J. H. Chung et al., "The soft supersymmetry-breaking lagrangian: Theory and applications," Phys. Rep., vol. 407, p. 1, 2005.

[3] CDF II Collaboration, Tech. Design Rep., FERMILAB-PUB-96390-E, 1996.

[4] A. Abulencia et al., The CDF II Extremely Fast Tracker Upgrade, FERMILAB-CONF-06-280-E, 2006.

[5] D. Cox, "CDF L2 track trigger upgrade," in Proc. 19th Hadron Collider Physics Symp., Galena, IL, May 27-31, 2008.

[6] A. Canepa et al., "The CDF level 2 calorimetric trigger," in Proc 10th Intern. Conf. Instr. Coll. Beam Physics, Novosibirsk, Russia, Feb./Mar. 2008.

[7] G. Cortiana, "An offline quality calorimetric selection for the CDF trigger," in Proc 10th ICATPP, Como, Italy, Oct. 2007.

[8] W. Ashmanskas et al., "The CDF silicon vertex tracker," Nucl. Instrum. Methods Phys. Res. A, vol. A518, pp. 532-536, Feb. 2004.

[9] A. Annovi et al., "A VLSI processor for fast track finding based on content addressable memories," IEEE Trans. Nucl. Sci., vol. 53, no. 4, pt. 2, pp. 2428-2433, Aug. 2006.

[10] T. Wright and D. Amidei, The CDF Collaboration, Search for Higgs Bosons Produced in Association With $b$-Quarks [Online]. Available: http://www-cdf.fnal.gov/physics/new/hdg/results/3b_susyhiggs_080229/cdf9284_higgs3b_v10.pdf

[11] P. W. Higgs, "Broken symmetries and the masses of gauge bosons," Phys. Rev. Lett., vol. 13, p. 508, 1964. 